Research articles

\title{
Exploring the electronic and magnetic properties of new metal halides from bulk to two-dimensional monolayer: $\operatorname{RuX}_{3}(\mathrm{X}=\mathrm{Br}, \mathrm{I})$
}

\author{
Fatih Ersan $^{\mathrm{a}, \mathrm{b}}$, Erol Vatansever ${ }^{\mathrm{c}}$, Sevil Sarikurt ${ }^{\mathrm{c}}$, Yusuf Yüksel ${ }^{\mathrm{c}}$, Yelda Kadioglua, ${ }^{\mathrm{a}}$, \\ H. Duygu Ozaydin ${ }^{\mathrm{a}}$, Olcay Üzengi Aktürk ${ }^{\mathrm{d}, \mathrm{e}}$, Ümit Akınc1 ${ }^{\mathrm{c}}$, Ethem Aktürk ${ }^{\mathrm{a}, e, *}$

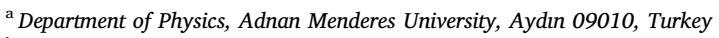 \\ ${ }^{\mathrm{b}}$ Department of Physics, Bilkent University, Ankara 06800, Turkey

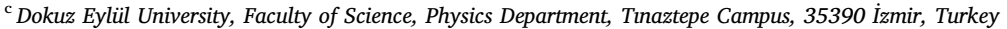 \\ ${ }^{\mathrm{d}}$ Department of Electrical and Electronic Engineering, Adnan Menderes University, 09100 Aydin, Turkey \\ ${ }^{\mathrm{e}}$ Nanotechnology Application and Research Center, Adnan Menderes University, Aydin 09010, Turkey
}

A R T I C L E I N F O

\section{Keywords:}

Monolayer $\mathrm{RuBr}_{3}$ and $\mathrm{RuI}_{3}$

Magnetic properties

Density functional theory

Monte Carlo method

\begin{abstract}
A B S T R A C T
Theoretical and experimental studies present that metal halogens in $\mathrm{MX}_{3}$ forms can show very interesting electronic and magnetic properties in their bulk and monolayer phases. Many $\mathrm{MX}_{3}$ materials have layered structures in their bulk phases, while $\mathrm{RuBr}_{3}$ and $\mathrm{RuI}_{3}$ have one-dimensional chains in plane. In this paper, we show that these metal halogens can also form two-dimensional layered structures in the bulk phase similar to other metal halogens, and cleavage energy values confirm that the monolayers of $\mathrm{RuX}_{3}$ can be possible to be synthesized. We also find that monolayers of $\mathrm{RuX}_{3}$ prefer ferromagnetic spin orientation in the plane for $\mathrm{Ru}$ atoms. Their ferromagnetic ground state, however, changes to antiferromagnetic zigzag state after $U$ is included. Calculations using PBE + U with SOC predict indirect band gap of $0.70 \mathrm{eV}$ and $0.32 \mathrm{eV}$ for the optimized structure of $\mathrm{RuBr}_{3}$ and $\mathrm{RuI}_{3}$, respectively. Calculation based on the Monte Carlo simulations reveal interesting magnetic properties of $\mathrm{RuBr}_{3}$, such as large Curie temperature against $\mathrm{RuI}_{3}$, both in bulk and monolayer cases. Moreover, as a result of varying exchange couplings between neighboring magnetic moments, magnetic properties of $\mathrm{RuBr}_{3}$ and $\mathrm{RuI}_{3}$ can undergo drastic changes from bulk to monolayer. We hope our findings can be useful to attempt to fabricate the bulk and monolayer of $\mathrm{RuBr}_{3}$ and $\mathrm{RuI}_{3}$.
\end{abstract}

\section{Introduction}

Recently, the family of transition metal trihalides $\mathrm{MX}_{3}$, where $\mathrm{M}$ is a metal cation ( $\mathrm{M}=\mathrm{Ti}, \mathrm{V}, \mathrm{Cr}, \mathrm{Fe}, \mathrm{Mo}, \mathrm{Ru}, \mathrm{Rh}, \mathrm{Ir})$ and $\mathrm{X}$ is halogen anion $(\mathrm{X}=\mathrm{Cl}, \mathrm{Br}, \mathrm{I})$, have received increasing attention due to their potential applications in spintronics [1-7]. Even though these materials have been known for more than 50 years [8-11], and their structure is wellinvestigated; only a few three-dimensional (3D) layered transition metal halides have been observed experimentally [12,13]. In recent years, it is possible to exfoliate these 3D layered crystals down to twodimensional (2D) monolayers, due to the weak interlayer van der Waals (vdW) interactions [14,15]. For instance, Weber et al. [16] report the exfolation of the magnetic semiconductor $\alpha-\mathrm{RuCl}_{3}$ into the first halide monolayers and investigations of its in-plane structure show that it is retained during the exfolation process. Huang et al. use magneto-optical Kerr effect (MOKE) microscopy to demonstrate the monolayer $\mathrm{CrI}_{3}$ is an Ising model ferromagnet (FM) with out-of-plane spin orientation. They find out that its Curie temperature of $45 \mathrm{~K}$ is only slight lower than $61 \mathrm{~K}$ of the bulk crystal [17], consistent with a weak interlayer coupling [18]. Similarly very recently, McGuire et al. [19] both experimentally and theoretically focus on the crystallographic and magnetic properties of transition metal compound $\alpha-\mathrm{MoCl}_{3}$ behavior above the room temperature.

Transition metal trihalides provide a rich family of materials with a wide range of electronic, optical and mechanical properties in which also low dimensional magnetism can be examined, and therefore rapidly increasing theoretical researches exists on this area [20-24]. In our previous study, we systematically investigate the electronic and magnetic properties of an $\alpha-\mathrm{RuCl}_{3}$ monolayer using density functional theory (DFT) and Monte Carlo (MC) simulations [25], and our cleavage energy calculations give smaller value than that of graphite, which means that the $\alpha-\mathrm{RuCl}_{3}$ monolayer can be easily obtained from its bulk phase and also we find that it is stable 2D intrinsic ferromagnetic semiconductor. Similarly, a class of $2 \mathrm{D}$ ferromagnetic monolayers $\mathrm{CrX}_{3}$

\footnotetext{
* Corresponding author at: Department of Physics, Adnan Menderes University, Aydın 09010, Turkey.

E-mail addresses: umit.akinci@deu.edu.tr (Ü. Akınc1), ethem.akturk@adu.edu.tr (E. Aktürk).
} 
( $\mathrm{X}=\mathrm{Cl}, \mathrm{Br}, \mathrm{I}$ ) is studied by Liu et al. [26] by using first-principle calculations combined with MC simulations based on the Ising Model. They confirm that the feasibility of exfoliation from their layered bulk phase by the small cleavage energy and all the ground states monolayers are semiconducting with band gaps of 2.28, 1.76 and $1.09 \mathrm{eV}$ for $\mathrm{CrCl}_{3}, \mathrm{CrBr}_{3}, \mathrm{CrI}_{3}$, respectively. Furthermore, the estimated Curie temperatures for $\mathrm{CrCl}_{3}, \mathrm{CrBr}_{3}, \mathrm{CrI}_{3}$ are found 66, 86, $107 \mathrm{~K}$, respectively. Different from this study, among the chronium trihalides, the $\mathrm{CrI}_{3}$ is also studied by another group both in experimentally and theoretically [27] since it is the simplest to prepare due to iodine can be handled relatively easy solid at room temperature. They find that an easily cleavable, layered and insulating ferromagnet with Curie temperature of $61 \mathrm{~K}$. Similarly, Huang et al. [28] examine $\mathrm{RuX}_{3}(\mathrm{X}=\mathrm{Cl}, \mathrm{Br}, \mathrm{I})$ monolayers and use only $\mathrm{RuI}_{3}$ monolayer as an exemplary material to study their electronic and magnetic properties by using first-principle calculations. Their result reveal that the ground state of the $\mathrm{RuI}_{3}$ monolayer is ferromagnetic with estimated Curie temperature to above the room temperature $\sim 360 \mathrm{~K}$. Nevertheless, $a b$ initio molecular dynamics (AIMD) simulations confirm its thermal stability at $500 \mathrm{~K}$ and also a clear Dirac cone in the spin-down channel appears at the $K$-point in the Brillouin zone near the Fermi level of its band structure. Similarly, relying on our previous experience [25], in this work we both focus on from bulk to monolayer $\mathrm{RuI}_{3}$ and $\mathrm{RuBr}_{3}$ electronic and magnetic properties in detail. Our results which are systematically investigated below are incompatible with the previous study. Since, our theoretical results demonstrate that $\mathrm{RuBr}_{3}$ and $\mathrm{RuI}_{3}$ can be stable in bulk form and monolayers of them can be obtained from their bulk phases by cleavage methods. We have obtained the possible magnetic ground state for bulk and monolayer forms of $\mathrm{RuBr}_{3}$ and $\mathrm{RuI}_{3}$ using $\mathrm{PBE}, \mathrm{PBE}+\mathrm{SOC}$ and $\mathrm{U}+\mathrm{SOC}$ calculations. The FM spin orientation is the most favorable configuration for PBE and PBE + SOC results, while zigzag spin orientation is favored after adding the Hubbard parameter. Hence, with considering the Hubbard $U$ correction, the favored spin orientation can be altered to antiferromagnetic zigzag situation and these $\mathrm{RuX}_{3}$ monolayer structures have suitable band gaps for various optoelectronic device applications. Afterwards, we have obtained the magnetic exchange coupling constants and magnetic anisotropy energies from the density functional theory calculations. Using these parameters, we have also performed Monte Carlo simulations, and estimated the Curie temperatures for $\mathrm{RuBr}_{3}$ and $\mathrm{RuI}_{3}$. According to our MC data, both structures in bulk and monolayer forms are found to be magnetically ordered at temperatures well below the room temperature.

\section{Model and formulation}

Density functional theory calculations were performed by using Vienna ab initio Simulation Package (VASP) $[29,30]$ within generalized gradient approximation (GGA) [31]. The Perdew-Burke-Ernzerhof (PBE) functionals were used for the exchange-correlation potential [32] and the Projector Augmented Wave (PAW) pseudo potentials were adopted [33,34]. A cutoff energy of $400 \mathrm{eV}$ for the plane wave basis set was used. Monkhorst-Pack [35] mesh of $12 \times 6 \times 6$ (for bulk) and $20 \times 10 \times 1$ (for monolayer) were employed for the Brillouin zone integration. A supercell with a $24 \AA$ vacuum distance was used in order to avoid interactions between two adjacent monolayer system when the periodic conditions are employed. The geometrical configurations were optimized by fully relaxing the atomic structures, until HellmannFeynman forces acting on each atom is reduced to less than $0.002 \mathrm{eV} / \AA$. The convergence of the total energy is achieved until the energy difference between successive iteration steps are less than $10^{-5} \mathrm{eV}$. Phonon dispersion curves were obtained by PHONOPY code [36] for the $2 \times 2 \times 1$ supercell and displacement of $0.01 \AA$ from the equilibrium atomic positions. Finite temperature AIMD calculations within Verlet algorithm were performed for thermal stability test. We used Nose thermostat for the duration of 2 picoseconds (ps) at $500 \mathrm{~K}$ for
$3 \times 3 \times 1 \mathrm{RuX}_{3}(\mathrm{X}=\mathrm{Br}, \mathrm{I})$ supercells.

To elucidate the magnetic structure of $\mathrm{RuX}_{3}$, and the nearest, second-nearest, and third-nearest neighbor exchange-coupling parameters $\left(J_{1}, J_{2}\right.$ and $J_{3}$, respectively), we adapt the total energy values obtained from DFT calculations for different magnetic configurations to the Heisenberg Spin Hamiltonian:

$$
\begin{aligned}
\mathscr{H}= & -J_{1} \sum_{<i j>} \mathbf{S}_{i} \cdot \mathbf{S}_{j}-J_{2} \sum_{\ll i k \gg} \mathbf{S}_{i} \cdot \mathbf{S}_{k}-J_{3} \sum_{\ll<i l \gg>} \mathbf{S}_{i} \cdot \mathbf{S}_{l} \\
& +k_{x} \sum_{i}\left(S_{i}^{x}\right)^{2}+k_{y} \sum_{i}\left(S_{i}^{y}\right)^{2},
\end{aligned}
$$

where $\mathbf{S}_{i}$ is the spin at the Ru site $\mathrm{i}$ and (i, j), (i, k) and (i, l) stand for the nearest, second-nearest and third-nearest Ru atoms, respectively. And $k_{x}$ and $k_{y}$ denote the out-of-plane magnetic anisotropy constants, respectively. The numerical values of $k_{x}$ and $k_{y}$ are obtained from magneto-crystalline anisotropy energies (MAE).

By mapping the DFT energies to the Heisenberg Hamiltonian, $J_{1}, J_{2}$ and $J_{3}$ can be calculated from following equations [37]:

$E_{F M / N e e l}=E_{0}-\left( \pm 3 J_{1}+6 J_{2} \pm 3 J_{3}\right) S^{2}$

and

$E_{\text {Zigzag } / \text { Stripy }}=E_{0}-\left( \pm J_{1}-2 J_{2} \mp 3 J_{3}\right) S^{2}$

The calculated $J_{1}, J_{2}$ and $J_{3}$ exchange-coupling parameters, in-plane (E[100]-E[010]) and out-of-plane (E[100]-E[001]) MAEs and magnetic anisotropy constants can be found in Supplementary Material (S.M) Tables S3-S5 for both $\mathrm{RuBr}_{3}$ and $\mathrm{RuI}_{3}$. While performing MAE calculations via DFT, we used Gamma-centered Monkhorst-Pack special k-point grids of $16 \times 8 \times 8$ and $24 \times 12 \times 1$ for bulk and monolayer structures, respectively. The Curie temperature was calculated by using these exchange-coupling parameters in MC simulations based on the Heisenberg model.

\section{Results and discussion}

\subsection{From bulk to two-dimensional monolayer $R u X_{3} ; D F T$ calculations}

Transition metal halides can be observed in several types of space groups such as $\mathrm{C} 2 / \mathrm{m}, \mathrm{Pmnm}, \mathrm{P}_{3} / \mathrm{mcm}, \mathrm{P} 3_{1} 12$. Among them metal halide crystal structure in $\mathrm{P} 3{ }_{1} 12$ space group has equidistant metal atoms in the cell. Experimentally $\mathrm{RuBr}_{3}$ can have Pmnm space group at low temperature while it has $\mathrm{P}_{3} / \mathrm{mcm}$ space group at high temperatures, and $\mathrm{RuI}_{3}$ has $\mathrm{P}_{3} / \mathrm{mcm}$ space group at room temperature [38]. In this paper we study only bulk $\mathrm{RuBr}_{3}$ and $\mathrm{RuI}_{3}$ structure in $\mathrm{P}_{1} 12$ space group, which is valid for $\mathrm{RuCl}_{3}$ (see Fig. 1). And also we obtain and investigate their stable monolayer forms. We initially constructed the bulk $\mathrm{RuBr}_{3}$ and $\mathrm{RuI}_{3}$ structures, and we obtained the optimized lattice constants as a $=6.25 \AA, b=10.83 \AA, c=6.31 \AA$ for $R^{2} B_{3}, a=6.77 \AA$, $\mathrm{b}=11.67 \AA$ and $\mathrm{c}=6.71 \AA$ for $\mathrm{RuI}_{3}$. Since they have not been sythesized in $\mathrm{RuCl}_{3}$ bulk type, we expose them in dynamical stability tests such as phonon and molecular dynamic (MD) calculations. Obtained phonon band structures are illustrated in Fig. 1(b). Phonon dispersions show that all phonon modes have positive value in the whole Brillouin zone, which imply the dynamical stability of bulk $\mathrm{RuX}_{3}(\mathrm{X}=\mathrm{Br}, \mathrm{I})$ structures in $\mathrm{P} 3_{1} 12$ space group. The thermodynamic variables of their bulk and monolayer phases are given in S.M. Fig. S1, and from this figure, we can state that the heat capacities trends of both bulk and monolayer forms of $\mathrm{RuX}_{3}$ structures follow the Dulong-Petit limit after around $200 \mathrm{~K}$. The AIMD calculations also showed that bulk form of $\mathrm{RuX}_{3}$ structures are thermally stable at $500 \mathrm{~K}$ for $2 \mathrm{ps}$. After optimization and stability calculations we examine their electronic properties, according to standard PBE calculations we found that both of bulk $\mathrm{RuX}_{3}$ structures are metal. Bader charge analysis indicates that each $\mathrm{Ru}$ atom in the bulk $\mathrm{RuBr}_{3}$ gives 0.70 electrons $\left(\mathrm{e}^{-}\right)$and each $\mathrm{Br}$ atom takes $0.23 \mathrm{e}^{-}$. These values are $0.30 \mathrm{e}^{-}$for $\mathrm{Ru}$ atoms and $0.10 \mathrm{e}^{-}$for I atoms in the bulk $\mathrm{RuI}_{3}$ structure. To examinate the favorable spin oriented status 


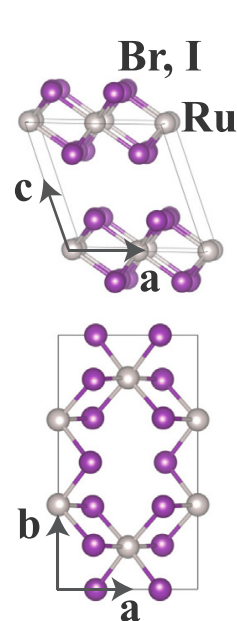

(a)

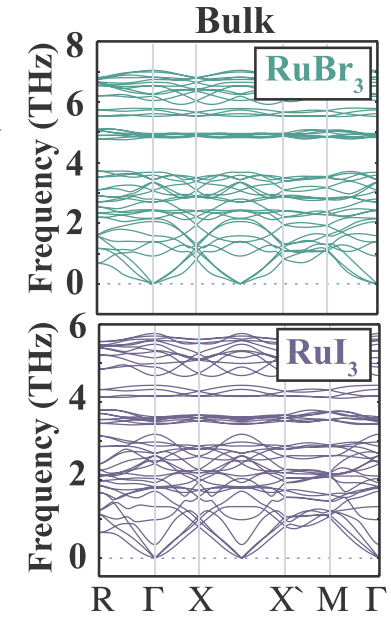

(b)

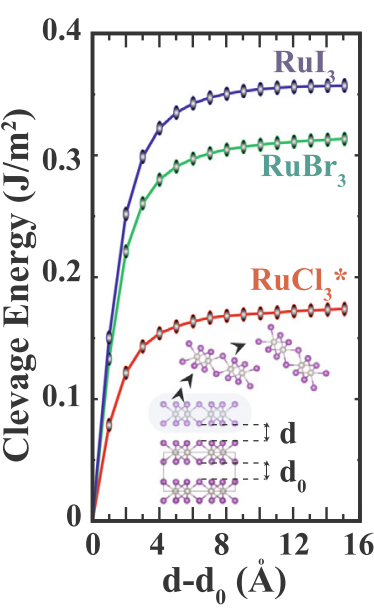

(c)

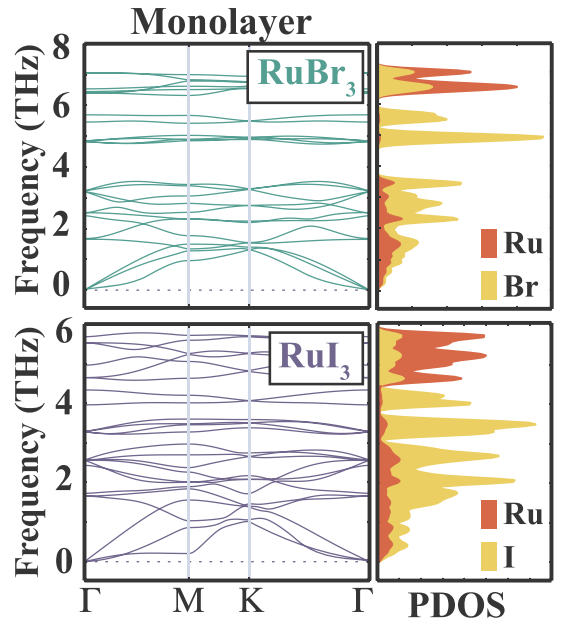

(d)

Fig. 1. a) Top and side view of bulk ( $\left(3_{1} 12\right.$ space group) $\mathrm{RuX}_{3}(\mathrm{X}=\mathrm{Br}, \mathrm{I})$ structures b) Phonon band structure of bulk RuX $\mathrm{X}_{3}$ structures. c) Cleavage energy as a function of separation between the two fractured parts. The fracture distance is denoted as $d$ and the equilibrium interlayer distance of ruthenium trihalides as $d_{0}$. Inside the graph: side view of bulk a- $\mathrm{RuX}_{3}$ used to simulate the exfoliation procedure, $\mathrm{RuCl}_{3}$ results taken from Sarikurt et al. study [25]. $\mathrm{d}$ ) Phonon band structures and corresponding PDOS of hexagonal $\mathrm{RuX}_{3}$ monolayer structures.

(a) FM

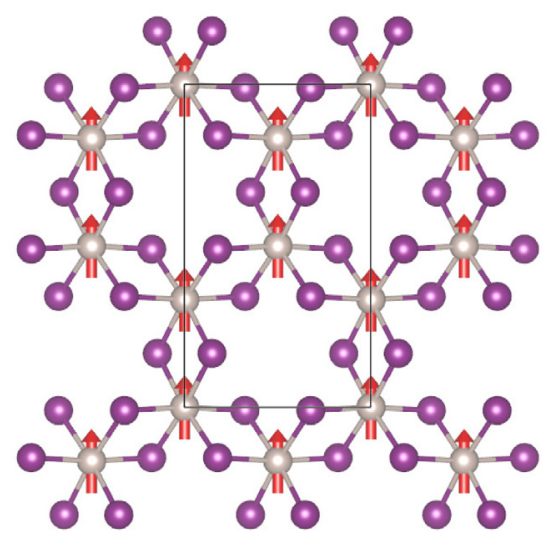

(c) AFM-Zigzag

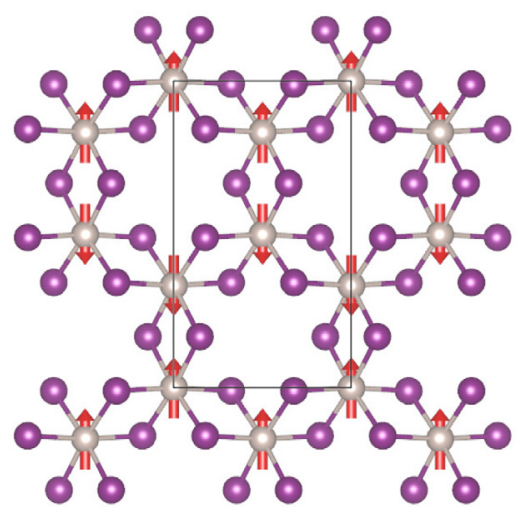

(b) AFM-Neel

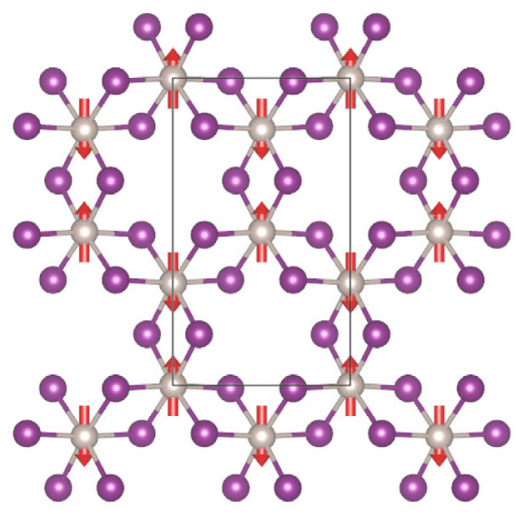

(d) AFM-Stripy

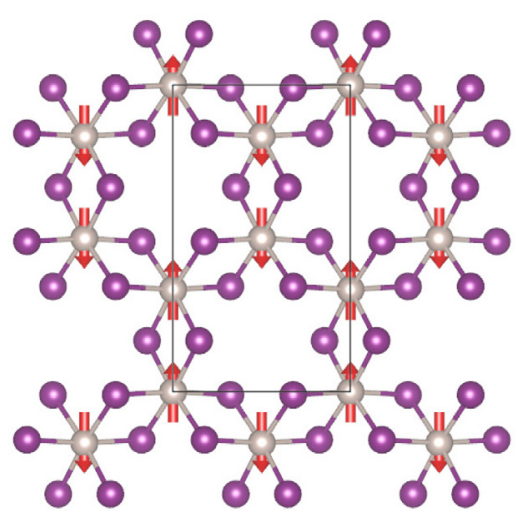

Fig. 2. Different spin configurations of the RuX 3 structures: (a) FM ordered, (b) AFM-Neél ordered, (c) AFM-Zigzag ordered and (d) AFM-stripy ordered.

in the bulk $\mathrm{RuX}_{3}$ structures four types of spin configurations are considered (FM, antiferromagnetic (AFM)-Neél, AFM-Zigzag and AFMStripy) for ruthenium atoms as seen in Fig. 2. We performed these calculations for three different DFT methods such as PBE, PBE + SOC (spin-orbit coupling) and $\mathrm{U}+\mathrm{SOC}$ (for Hubbard $\mathrm{U}=1.5 \mathrm{eV}$ ) calculations. According to the calculations FM spin orientation is favorable for $\mathrm{PBE}$ and $\mathrm{PBE}+\mathrm{SOC}$ results, while zigzag spin orientation is favored after adding the Hubbard parameter (please see S.M Table S1 for 
relative energies, and band structures of bulk $\mathrm{RuX}_{3}$ ). Finally, we tested the possibility of the exfoliation techniques to get few layers of monolayer from their bulk forms. For these calculations bulk $\mathrm{RuX}_{3}$ structures are extended in $\mathrm{z}$-direction and four layered $\mathrm{RuX}_{3}$ structures are created, and then we implemented a fracture in the bulk after four periodic layers and systematically increased this fracture distance; at the end we calculated the corresponding cleavage energy (CE) (Fig. 1). $\mathrm{RuCl}_{3}$ results are taken from our previous study [25]. As can be seen increasing of the halogens rows in the periodic table enhances the cleavage energy. But calculated energies are comparable with graphite, and other $\mathrm{MX}_{3}$ materials $[3,8,20,21,26,27,39]$.

Monolayer $\mathrm{RuBr}_{3}$ and $\mathrm{RuI}_{3}$ structures are constructed in hexagonal unitcell, which have lattice constants of $\mathrm{a}=6.25 \AA$, and $\mathrm{a}=6.78 \AA$ for $\mathrm{RuBr}_{3}$ and $\mathrm{RuI}_{3}$, respectively. This lattice value is $5.92 \AA$ for $\mathrm{RuCl}_{3}$ [25] as expected lattice constants increase by increasing the atomic radii from chlorine to iodine. We could not compare the results of $\mathrm{RuBr}_{3}$ and $\mathrm{RuI}_{3}$ with experimental data due to lack of experimental study to the best of our knowledge. Pauling electronegativity values are 2.20 for $\mathrm{Ru}$ atom 2.96 for $\mathrm{Br}$ and 2.66 for I atoms, this electronegativity difference results more electron transferring from $\mathrm{Ru}$ atoms to $\mathrm{Br}$ atoms than I atoms. According to Bader charge analysis while each $\mathrm{Ru}$ atom in $\mathrm{RuBr}_{3}$ loses 0.72 electrons $\left(\mathrm{e}^{-}\right)$, this value is $0.32 \mathrm{e}^{-}$in $\mathrm{RuI}_{3}$ monolayer. This charge transfer interpretable such as there is more strength bond between $\mathrm{Ru}$ and $\mathrm{Br}$ atoms according to Ru-I atoms. Similar dynamical tests which are performed for bulk $\mathrm{RuX}_{3}$ structures are also performed for monolayers. Phonon band structures and their partial density of states (PDOS) of $\mathrm{RuX}_{3}$ monolayers are illustrated in Fig. 1. Phonon dispersions are obtained by using PHONOPY code which is based on density functional perturbation theory as implemented in VASP. As can be seen, all phonon branches have positive frequency values in the whole Brillouin Zone (BZ) which implies the dynamical stability at $\mathrm{T} \sim 0 \mathrm{~K}$. As mentioned later, spin-polarization is more effective in $\mathrm{RuBr}_{3}$ with respect to $\mathrm{RuI}_{3}$ monolayer. Thus, phonon band structure of $\mathrm{RuBr}_{3}$ obtained with spin-polarized calculation due to it has large imaginary frequencies for out-of-plane acoustical branch (ZA) for spin-unpolarized status. In addition, phonon band structure of $\mathrm{RuI}_{3}$ monolayer has a local minimum at the M high symmetry point for the ZA, which is associated with Kohn anomalies. Thermal stability tests are performed by AIMD calculations. All $\mathrm{RuX}_{3}$ structures subjected to $500 \mathrm{~K}$ temperature for 2 ps. At the end of calculations both of $\mathrm{RuBr}_{3}$ and $\mathrm{RuI}_{3}$ monolayers preserved their optimized atomic configuration which are obtained at $\mathrm{T}=0 \mathrm{~K}$ calculations. This means that $\mathrm{RuX}_{3}$ monolayers can be stable at room temperature and at least slightly above it. This conclusion is very important to utilize them in device technology. After the stability tests we start to investigate to determine their favorable magnetic ground states. For this examination, we changed the hexagonal $\mathrm{RuX}_{3}$ unitcell to the rectangular cell and considered four types of spin configurations similar to bulk ones as seen in Fig. 2. We performed geometric optimization calculations to the structures for all considered magnetic orientation status until the pressure on the cell is approximately zero, with and without spin-orbit coupling effect. According to these PBE and $\mathrm{PBE}+\mathrm{SOC}$ calculations we found that FM state is energetically favorable spin oriented status for both $\mathrm{RuBr}_{3}$ and $\mathrm{RuI}_{3}$ monolayers. But AFMStripy- $\mathrm{RuBr}_{3}$ has only $86 \mathrm{meV}$ higher energy than FM state and this difference is reduced to $67 \mathrm{meV}$ when the SOC is added in calculations. SOC is more effective in $\mathrm{RuI}_{3}$ monolayer, energy difference between FM and Stripy state is $119 \mathrm{meV}$ without SOC effects, while it becomes $10 \mathrm{meV}$ with SOC contribution. Relative energy differences for other spin orientation states can be found in S.M Table S2. Each FM-RuX structure has $4 \mu_{B}$ magnetic moment in per rectangular cell and each Ru atom in the cell has $1 \mu_{B}$ magnetic moment. We also calculated the cohesive energies of FM-RuX 3 structures to determine the strength of cohesion between the Ru and $\mathrm{X}$ atoms and we estimate $13.67 \mathrm{eV}$ and $12.81 \mathrm{eV}$ for per $\mathrm{RuBr}_{3}$ and $\mathrm{RuI}_{3}$ quartet atoms, respectively. Dominant orbital contribution to the electronic structure comes from $\mathrm{Ru} d$ and halogen $p$ orbitals, Fig. 3 shows the electronic PDOS of $\mathrm{RuX}_{3}$ monolayers for various spin-orientation and with (w) and without (w/ o) SOC effect. As seen in Fig. 3 a) both of monolayers have large band gap for spin up channel, ordered in $1.65 \mathrm{eV}$ and $1.45 \mathrm{eV}$ for $\mathrm{FM}-\mathrm{RuBr}_{3}$ and $\mathrm{FM}-\mathrm{RuI}_{3}$, while density of states are very close to each other for spin down channels (There is a $20 \mathrm{meV}$ gap between the DOSs for FM$\mathrm{RuBr}_{3}$, while this gap reaches to $100 \mathrm{meV}$ for $\mathrm{FM}-\mathrm{RuI}_{3}$ ). For FM-RuBr spin up state, two-fold $e_{g}\left(d_{z^{2}}\right.$ and $\left.d_{x^{2}}-d_{y^{2}}\right)$ orbitals and three-fold $t_{2 g}$ $\left(d_{x y}, d_{y z}\right.$ and $\left.d_{x z}\right)$ orbitals contribute equally to the valence band maximum (VBM), while there are just $t_{2 g}$ orbitals contribution in conduction band minimum (CBM) and between 1.7 and $1.9 \mathrm{eV}$. Also dominant contribution comes from $t_{2 g}$ orbitals for spin down channel around the Fermi level, and again there are only $t_{2 g}$ orbitals between 1.8 and $2.0 \mathrm{eV}$ in spin down. $\mathrm{Br}$ atom $p$ orbitals give approximately equal contribution around VBM and CBM for both spin up and down channels (see S.M Fig. $\mathrm{S} 3$ ). For $\mathrm{FM}-\mathrm{RuI}_{3}$ spin up two-fold $e_{\mathrm{g}}$ orbitals are dominant at VBM and at CBM, $t_{2 g}$ orbitals contributions start $\sim 0.2 \mathrm{eV}$ lower energy from VBM, while there are not in $\mathrm{CB}$. Spin down states posses similar situation with $\mathrm{FM}-\mathrm{RuBr}_{3}$ spin down channel. In plane $p$ orbitals $\left(p_{x}, p_{y}\right)$ of iodine give major contribution to the VBM for spin up state as seen in Fig. S3. By including SOC effect in calculation for $\mathrm{FM}-\mathrm{RuBr}_{3}$ system gains metallic

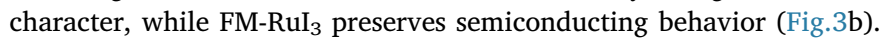
Electric and magnetic properties of such layered metal halides must be investigated by including Hubbard $U$ correction term to the calculations, so we added $U$ from 0.5 to $3.0 \mathrm{eV}$ which increases by successive $0.5 \mathrm{eV}$ value and we determined the favorable magnetic ground states for each added $U$ terms, we also repeated these calculations by adding $U+$ SOC terms in our calculations. Relative ground state energy graphs can be found in S.M. Fig. S4. According to our extended calculations, FM spin orientation is favorable just for $\mathrm{U}=0.5 \mathrm{eV}$ both with and without SOC effect. For larger Hubbard energies zigzag (ZZ) orientation has minimum ground state energy comparing to others. We attained very close band gap value with experimentally obtained thin layered $\alpha-\mathrm{RuCl}_{3}$ result [40] in our previous band structure calculations for $\mathrm{RuCl}_{3}$ monolayer for Hubbard $\mathrm{U}=1.5 \mathrm{eV}$, thus we give in detail density of states for energetically favorable $\mathrm{ZZ}-\mathrm{RuX} \mathrm{X}_{3}(\mathrm{U}+\mathrm{SOC}$ and $\mathrm{U}=1.5 \mathrm{eV})$ monolayers in Fig. 3c. As can be seen in Fig. 3c Hubbard U and SOC effects enhance the band gaps for $\mathrm{RuX}_{3}$ monolayers and reaches $0.70 \mathrm{eV}$ for $\mathrm{RuBr}_{3}$ and $0.32 \mathrm{eV}$ for $\mathrm{RuI}_{3}$. While $t_{2 g}$ orbitals of Ru atoms and $p_{x}, p_{y}$ orbitals of I atoms determine the VBM level, all orbitals of $\mathrm{Ru}$ and $\mathrm{Br}$ atoms approximately give similar contribution at VBM. At conduction band minimums $t_{2 g}$ orbitals of $\mathrm{Ru}$ atoms are dominant. Calculated electronic band structures for all optimized $\mathrm{RuX}_{3}$ monolayers, and also band trends can be found in S.M Figs. S5-S7.

\subsection{From bulk to two-dimensional monolayer $\mathrm{RuX}_{3}$; Monte Carlo calculations}

\subsubsection{Heisenberg-Kitaev models}

Recently, magnetic properties of certain materials exhibiting strong SOC have been modeled by using the Heisenberg-Kitaev (HK) model [41-44]. For instance, magnetic behaviors of $\alpha-\mathrm{RuCl}_{3}$ and $\mathrm{Na}_{2} \mathrm{IrO}_{3}$ have been studied by Janssen et al. [45]. They have demonstrated that the response of the system to an external field differs substantially for the different scenarios of stabilizing the zigzag state. The same group have also studied the honeycomb lattice HK model in an external magnetic field, and mapped out the classical phase diagram for different directions of the magnetic field [46]. In addition, magnetic behavior and phase diagrams of iridium oxides $\mathrm{A}_{2} \mathrm{IrO}_{3}$ have been investigated by Chaloupka and coworkers $[47,48]$ and by Singh et al. [49]. The latter group have demonstrated that the magnetic properties of $\mathrm{A}_{2} \mathrm{IrO}_{3}$ can be modeled by using $\mathrm{HK}$ model including next-nearest neighbor interactions.

Apart from these works, there also exist several works dedicated to the investigation of magnetic properties of HK model in detail. For instance, the topological properties of the expanded classical HK model on a honeycomb lattice have been investigated by Yao and Dong [50]. 


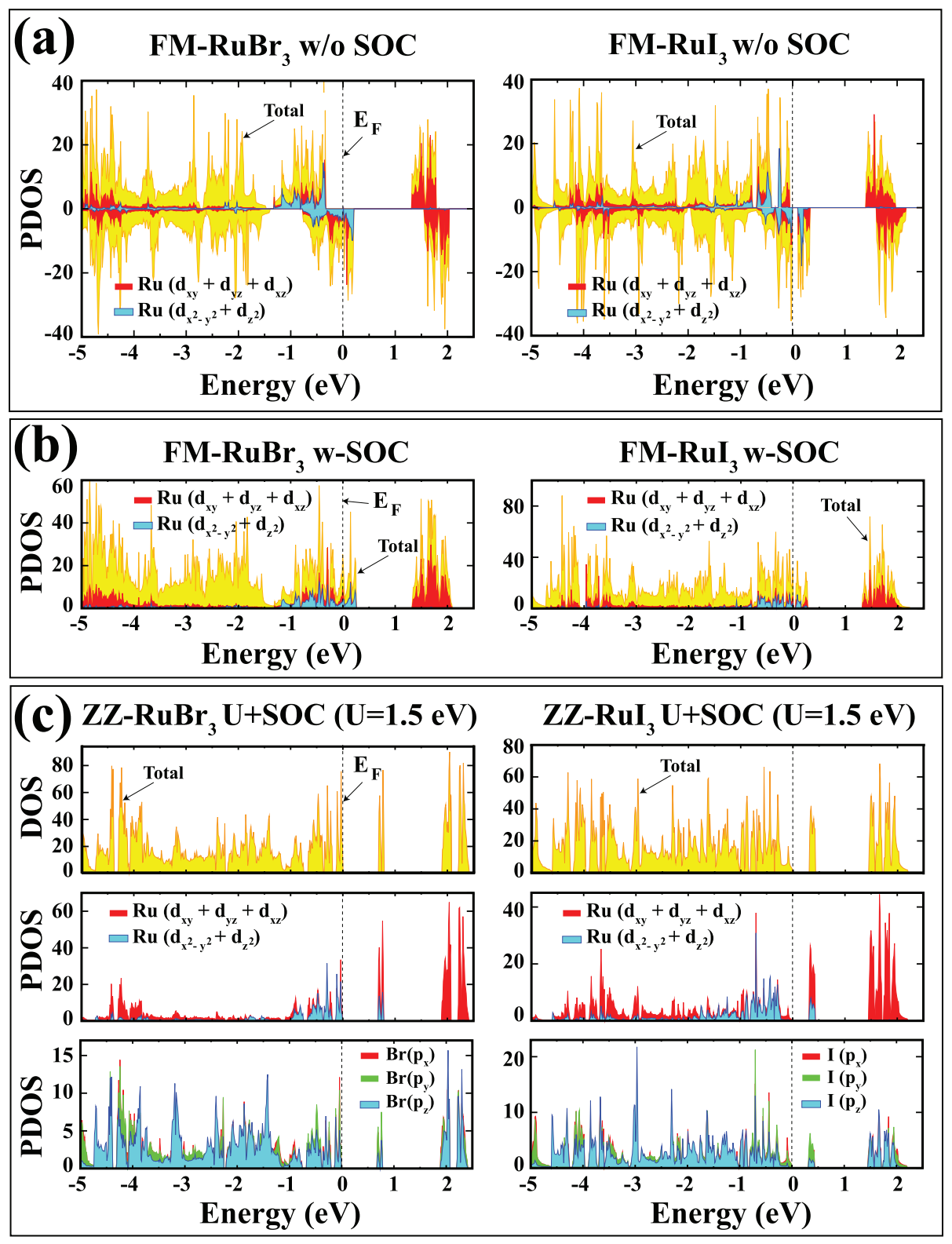

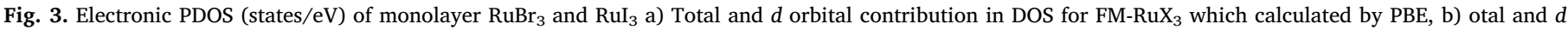

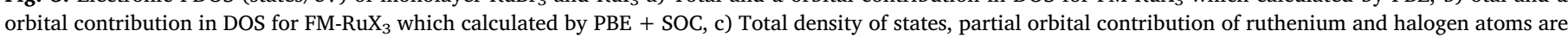
given separately for $\mathrm{ZZ}-\mathrm{RuX}_{3}$ which is calculated with Hubbard $\mathrm{U}+\mathrm{SOC}(\mathrm{U}=1.5 \mathrm{eV})$ effect.

The effect of the spatially anisotropic exchange couplings on the orderdisorder characteristics of HK model has been clarified by Sela et al. [51]. Classical HK model on a triangular lattice including the nextnearest neighbor interactions and single ion anisotropy has been investigated by Yao [52]. Price and Perkins [53] elaborated the finite temperature phase diagram and order-disorder transitions of classical HK model on a hexagonal lattice. In a separate work [54], they have also studied the critical properties of the HK model on the honeycomb lattice at finite temperatures in which they have found that the model undergoes two phase transitions as a function of temperature. Finally, the relation between the classical HK model and quantum spin- $S$ Kitaev model for large $S$ has been discussed by Chandra et al. [55].

Although Ising model is often utilized in determination of magnetic properties of real magnetic materials [28], one may desire to take into account the apparent effect of MAE (see S.M Table S4) in the atomistic spin model calculations. Hence, for simplicity, we base our simulations on an anisotropic Heisenberg model. Although, classical Heisenberg model is a simple model in comparison to HK model, it provides physically more reasonable results in comparison to conventional Ising model which is only suitable for highly anisotropic magnetic systems.

\subsubsection{Monte Carlo simulation details}

In order to clarify the magnetic properties of $\operatorname{RuX}_{3}(\mathrm{X}=\mathrm{Br}, \mathrm{I})$, we proposed an atomistic spin model, and performed MC simulations based on the Metropolis algorithm [56] on a two dimensional honeycomb lattice with lateral dimensions $L_{x}=L_{y}=100$ which contains $N=10^{4}$ spins. We run our simulations based on the Hamiltonian defined by Eq. (1). The numerical values of system parameters have been provided in S.M Tables S3 and S4. According to Eq. (1), a Ru atom with a pseudo spin $\left|\mathbf{S}_{i}\right|=1 / 2$ resides on each lattice site. We can briefly outline the simulation procedure based on Eq. (1) as follows: Starting from a high temperature spin configuration, we progressively cool down the system until the temperature reaches $T=10^{-2} \mathrm{~K}$. We performed sequential spin flip update in our calculations with $10^{5} \mathrm{MC}$ steps per site where $10 \%$ of this value have been discarded for thermalisation. Periodic boundary conditions (PBC) were imposed in all directions. In order to reduce the 
statistical errors, we performed 100 independent runs at each temperature. Error bars were calculated using the Jackknife method [56]. During the simulation, the following physical properties have been monitored:

- Time series of the spatial components of total magnetization

$$
m_{\alpha}(t)=\frac{1}{N} \sum_{i=1}^{N} g \mu_{B} S_{i}^{\alpha}, \quad \alpha=x, y, z
$$

where $g$ is the Landé factor, and $\mu_{B}$ is the Bohr magneton. Using Eq. (4), we can obtain the thermal average of the magnitude of the total magnetization vector $M_{T}$, as well as its components $M_{\alpha}$ according to the following relations

$$
\left\langle M_{\alpha}\right\rangle=\left\langle m_{\alpha}(t)\right\rangle, \quad\left\langle M_{T}\right\rangle=\left\langle\sqrt{\sum_{\alpha=x, y, z} m_{\alpha}^{2}(t)}\right\rangle .
$$

- In order to locate the transition temperature, we have also calculated the thermal average of magnetic susceptibility $\chi$ and magnetic specific heat as follows

$$
\chi=N\left(\left\langle M_{T}^{2}\right\rangle-\left\langle M_{T}\right\rangle^{2}\right) / k_{B} T,
$$

$C=N\left(\left\langle\mathscr{H}^{2}\right\rangle-\langle\mathscr{H}\rangle^{2}\right) / k_{B} T^{2}$.

where $k_{B}$ is the Boltzmann's constant. For the sake of completeness, we have also calculated the specific heat via

$C=\frac{\partial\langle\mathscr{H}\rangle}{\partial T}$.

\subsubsection{Monte Carlo simulation results}

In Fig. 4, we display the MC simulation results regarding the magnetic properties of simulated $\mathrm{RuX}_{3}(\mathrm{X}=\mathrm{Br}, \mathrm{I})$ monolayer systems. In Fig. 4a, we plot the magnetization versus temperature for both structures. As seen in this figure, starting from high temperature configuration, as the temperature gradually decreases then the non zero magnetization components emerge. Since the out-of-plane anisotropy constants $k_{x}$ and $k_{y}$ equal to each other, main contribution to the total magnetization equally comes from $x$ and $y$ components whereas $z$ component does not contribute to the magnetic behavior. Although the components exhibit apparent fluctuations in the considered temperature range, the magnitude $\left\langle M_{T}\right\rangle$ of total magnetization exhibits rather smooth behavior with error bars smaller than the data points. At very low temperatures $\left\langle M_{T}\right\rangle$ saturates to unity which means that $\mathrm{RuX}_{3}(\mathrm{X}=\mathrm{Br}, \mathrm{I})$ system exhibits ferromagnetic behavior at the ground state. This is consistent with results of our DFT calculations where we predicted that the stable ground state of $\operatorname{RuX}_{3}(\mathrm{X}=\mathrm{Br}, \mathrm{I})$ is FM. Thermal variation of internal energy is shown in Fig. 4b. Absolute value of $\langle\mathscr{H}\rangle$ at low temperature region is larger than that of the high temperature region. This is due to the fact that with increasing temperature, thermal fluctuations are enhanced, and the system evolves towards the paramagnetic regime. The transition temperature of $\mathrm{RuX}_{3}$ monolayers can be determined by examining the magnetic susceptibility and specific heat curves which are depicted in Fig. $4 \mathrm{c}$ and d. As seen in these figures, both response functions exhibit a peculiar peak in the vicinity of the magnetic phase transition temperature. According to our simulation results, transition temperature values separating the ferromagnetic phase from paramagnetic phase are found to be $T_{c}=2.11 \mathrm{~K}$ and $T_{c}=13.0 \mathrm{~K}$ for $\mathrm{RuI}_{3}$ and $\mathrm{RuBr}_{3}$, respectively. Relatively small $T_{c}$ for the former structure is a direct consequence of weak $J_{i}$ values of this structure (see S.M Tables S3 and S4). $T_{c}$ value obtained for $\mathrm{RuI}_{3}$ monolayer is reasonably below the value reported by Huang and coworkers [28]. The reason is straightforward based on two reasons. First, in Ref. [28], the authors considered only the nearest neighbor exchange interactions with $J_{1}=82 \mathrm{meV}$ which is fairly larger than our predicted value. Second, they omitted the effect of MAE (it seems that MAE is rather influential in $\mathrm{RuX}_{3}$, see Table S.M Table S4) in their calculations. On the other hand, $T_{c}$ value obtained for $\mathrm{RuBr}_{3}$ can be compared with $T_{c}=14.21 \mathrm{~K}$ for $\mathrm{RuCl}_{3}$ reported in our recent work [25]. We note that recently it has also been reported for $2 \mathrm{D}$ ferromagnetic monolayers $\mathrm{CrX}_{3}(\mathrm{X}=\mathrm{Br}, \mathrm{I})$ that Curie temperature of $\mathrm{CrBr}_{3}$ is lower than that obtained for $\mathrm{CrI}_{3}$ [26]. This is an opposite scenario in comparison to our reported values for $\mathrm{RuX}_{3}$ where the Curie temperature of $\mathrm{RuBr}_{3}$ is larger than that of $\mathrm{RuI}_{3}$. These results show that the presence of $\mathrm{Ru}$ instead of $\mathrm{Cr}$ in monolayer trihalides $\mathrm{MX}_{3}(\mathrm{X}=\mathrm{Br}, \mathrm{I})$ may cause dramatic differences incritical behavior of these structures. Moreover, as we mentioned before, based on our rigorous DFT calculations, we believe that the magnetic behavior of such systems cannot be modeled using standard Ising model, since the MAE plays a significant role in the magnetic behavior of these materials. Therefore we suggest to use the anisotropic Heisenberg model in atomistic spin model calculations. Fig. 5.

Apart from these observations, using the Hamiltonian parameters provided in S.M Tables S3 and S4, we have also performed MC simulations for the bulk $\operatorname{RuX}_{3}(\mathrm{X}=\mathrm{Br}, \mathrm{I})$. By assuming weak van der Waals bonding between adjacent magnetic interlayers $[7,15,57]$, we followed the same simulation procedure defined for our monolayer systems. According to our simulation data, we found that the transition temperatures for $\mathrm{RuI}_{3}$ and $\mathrm{RuBr}_{3}$ in bulk form are given as $T_{c}=0.11 \mathrm{~K}$ and $T_{c}=13.3 \mathrm{~K}$, respectively. We note that although the Curie temperature of monolayer $\mathrm{RuBr}_{3}$ is comparable to its bulk counterpart, the situation is different for $\mathrm{RuI}_{3}$ where the critical temperature of the bulk system is lower than that of the monolayer system. This is primarily due to the fact that while the values of the exchange interactions for monolayer and bulk cases are in the same order for $\mathrm{RuBr}_{3}$, the bulk exchange coupling parameters of $\mathrm{RuI}_{3}$ predicted by our DFT calculations have been found to be fairly weaker than those calculated for the monolayer case (c.f. S.M Table S3). This means that a small amount of thermal fluctuation can be enough to destroy the magnetic order for the bulk $\mathrm{RuI}_{3}$ system. Based on a recent experimental work [18], bulk to monolayer transition in $\mathrm{CrI}_{3}$ have been reported with respective transition temperatures $T_{c}=61 \mathrm{~K}$ (bulk) and $T_{c}=45 \mathrm{~K}$ (monolayer). From this point of view, we have an opposite scenario where our $\mathrm{RuI}_{3}$ system in bulk form exhibits lower critical temperature than that obtained for the monolayer limit. Hence, we can conclude that due to the presence of $\mathrm{Ru}$ instead of $\mathrm{Cr}$ in trihalides of the form $\mathrm{MX}_{3}(\mathrm{X}=\mathrm{Br}, \mathrm{I})$, the bulk magnetic properties may also be significantly altered. This can be a direct consequence of different spin magnitudes of Ru and $\mathrm{Cr}$, different exchange energies in the intralayer, as well as interlayer regions, etc. In conclusion, one cannot establish a general trend for the critical behavior (i.e. variation of the critical temperature with the spatial dimension) of $\operatorname{RuX}_{3}(\mathrm{X}=\mathrm{Br}, \mathrm{I})$ when the topology evolves from bulk to monolayer.

\section{Conclusion}

In conclusion, with the help of first-principles calculations we theoretically showed that bulk $\mathrm{RuBr}_{3}$ and $\mathrm{RuI}_{3}$ could be stable in $\mathrm{P} 3_{1} 12$ space group similar to $\alpha-\mathrm{RuCl}_{3}$. According to cleavage energy calculations, monolayer forms of $\mathrm{RuX}_{3}$ structures can be easily attained from their bulk phases. Also we tested dynamical and thermal stabilities of monolayers and found that they can be stable at room temperature and above. While ferromagnetic spin orientation is favorable state for PBE and $\mathrm{PBE}+\mathrm{SOC}$ calculations, Hubbard $\mathrm{U}$ and $\mathrm{U}+\mathrm{SOC}$ calculations show that AFM-zigzag cases have minimum ground state energies comparing to others except for $\mathrm{U}=0.5 \mathrm{eV}$. However, electronic band structures of all spin oriented configurations show similarities, $U$ and SOC effects enhance the band gaps. While $\mathrm{RuI}_{3}$ monolayer has band gap 
(a)

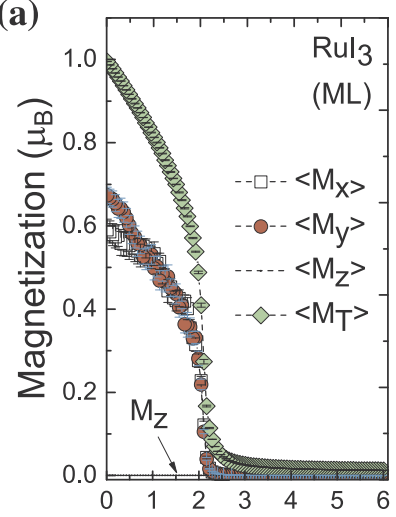

(c)

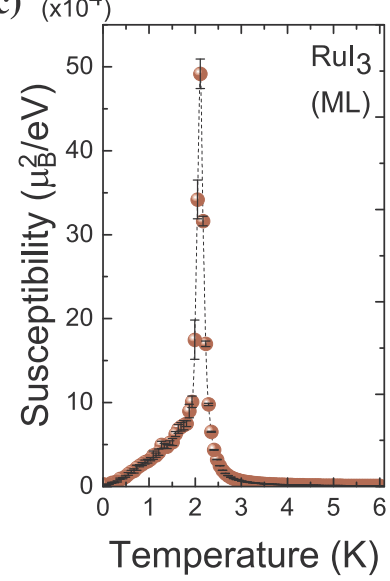

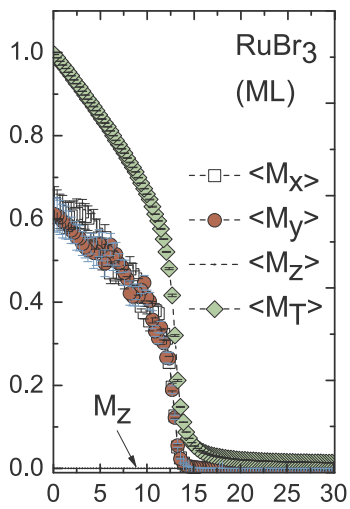
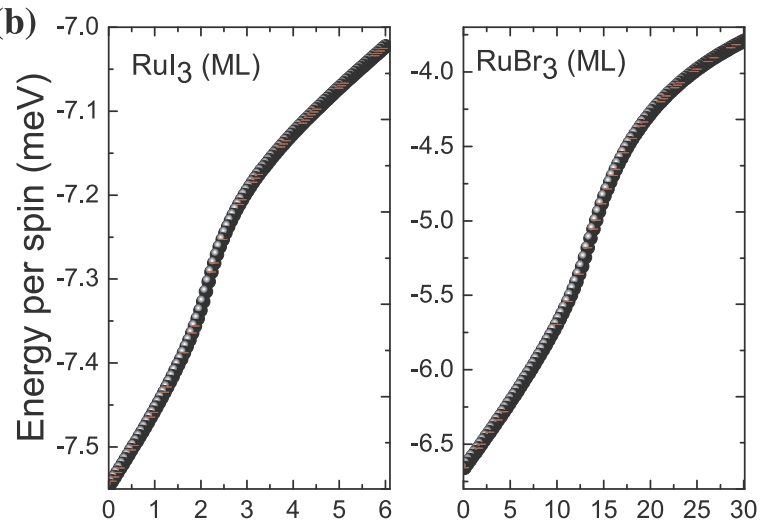

$\left(\times 10^{4}\right)$

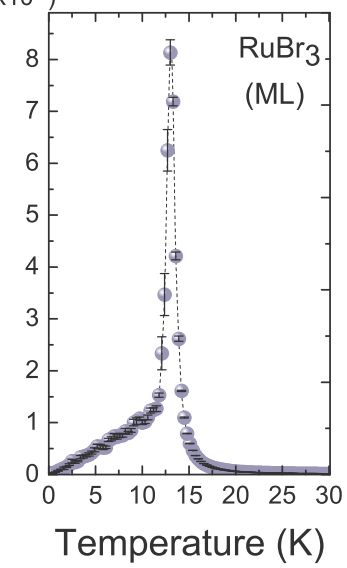

(d) $\left(\times 10^{-4}\right.$

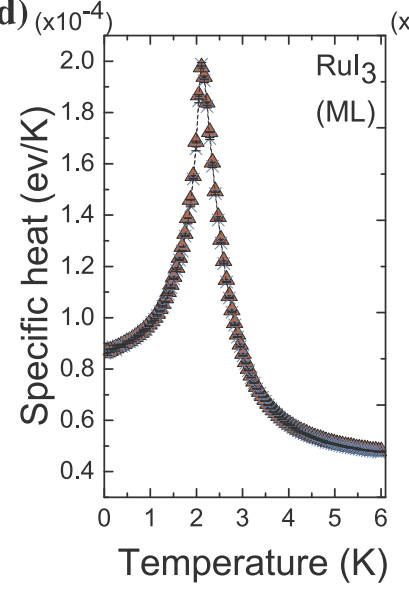

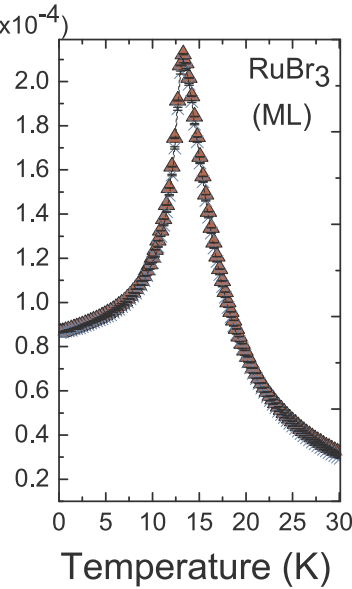

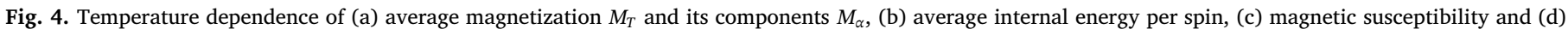

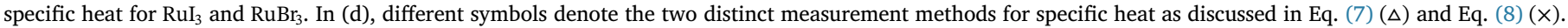

(a)

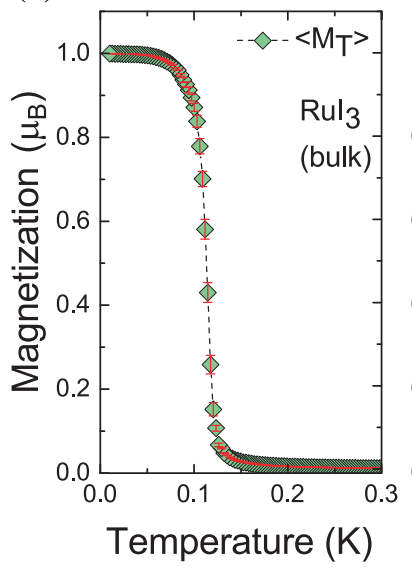

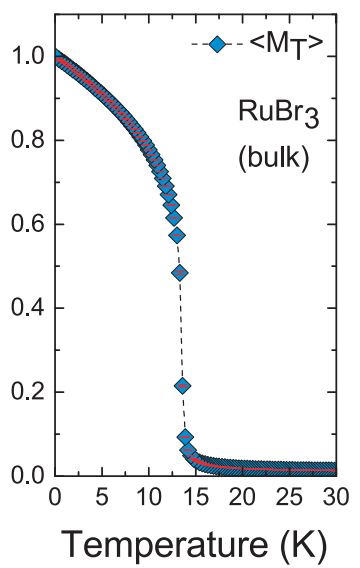

(b)
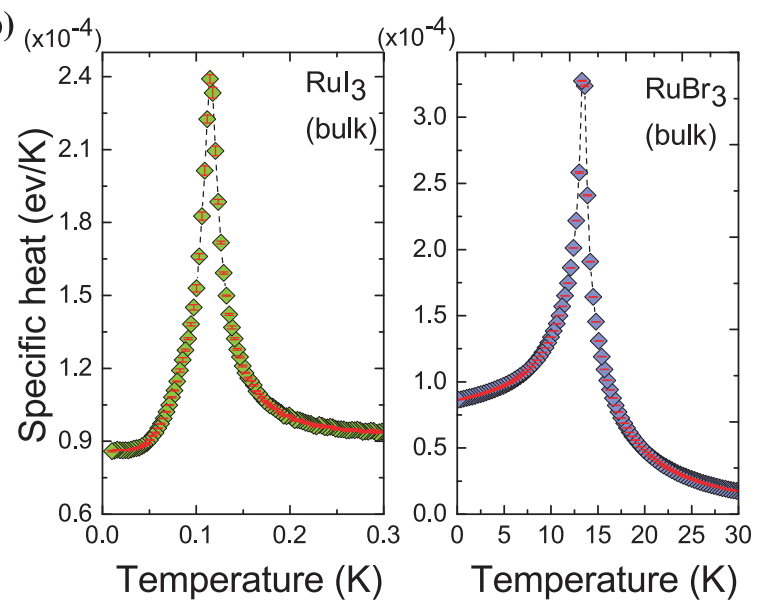

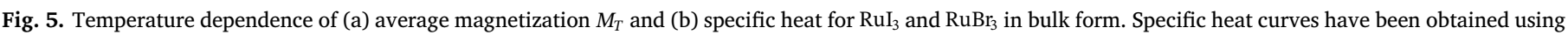
Eq. (8).

values in the range of infrared region, band gap values of $\mathrm{RuBr}_{3}$ monolayer can reach the near visible region according to spin orientation configuration and $\mathrm{U}$ parameter. We have also performed detailed Monte Carlo simulations to clarify the magnetic properties of $\mathrm{RuBr}_{3}$ and $\mathrm{RuI}_{3}$. Using the atomistic model parameters (i.e. exchange and magnetic anisotropy energies) obtained from PBE + SOC calculations, we have found that the Curie temperature of $\mathrm{RuBr}_{3}$ dominates against that of $\mathrm{RuI}_{3}$ both in bulk and monolayer forms. However, obtained critical temperature values are found to be far from the room temperature. Furthermore, some drastic changes may originate in the magnetic behavior of these systems when the form is changed from bulk to monolayer. According to the DFT calculations based on U + SOC, ground state configuration evolves from ferromagnetic to antiferromagnetic zigzag which causes prominent changes in the numerical values of simulation parameters (c.f. see S.M Tables S3, S6, S7). Besides, since the magnetic character of the first, second and third nearest neighbors turn into AFM type, $\mathrm{RuX}_{3}$ system in bulk and monolayer phases exhibits Neel temperature instead of Curie temperature. More importantly, frustration effects take place in the system which completely affects the magnetic behavior. We should also note that, by comparing the magnetic anisotropy constants in the presence of $\mathrm{U}+\mathrm{SOC}$, we see that in the monolayer case, absolute values of the 
anisotropy constants attain lower values, in comparison to the case of PBE + SOC. In addition, in the monolayer case, anisotropy constants also lose their in plane isotropy (c.f. compare the numerical values of $k_{x}$ and $k_{y}$ between S.M. Tables S4 and S5). Overall, the entire magnetic behavior of $\mathrm{RuX}_{3}(\mathrm{X}=\mathrm{Br}, \mathrm{I})$ may be highly sensitive to the consideration of Hubbard U parameter in DFT calculations. Such that according to $\mathrm{U}$ parameter, spin orientation and by this way the equilibrium structure can be changed. U parameter also can change the band gap values therefore electronic characteristics of systems. Last but not least, the Hamiltonian defined by Eq. (1) successfully explains the magnetic behavior (i.e. transition temperature and ground state magnetic order) of $\mathrm{RuCl}_{3}$. Besides, since $\mathrm{RuCl}_{3}$ and $\mathrm{RuX}_{3}(\mathrm{X}=\mathrm{Br}, \mathrm{I})$ are similar then it may be expected that our predictions based on Eq. (1) would successfully resemble the future experimental works on $\mathrm{RuX}_{3}$. We believe that this study can play an important role, for the future attempt to obtain bulk and monolayer forms of $\mathrm{RuBr}_{3}$ and $\mathrm{RuI}_{3}$.

\section{Acknowledgements}

This work was supported by the Scientific and Technological Research Council of Turkey (TÜBITAK) under the Research Project No. 117F133. Computing resources used in this work were provided by the TÜBİTAK ULAKBIM, High Performance and Grid Computing Center (TrGrid e-Infrastructure) and the National Center for High Performance Computing of Turkey (UHeM) under Grant No. 5004972017.

\section{Appendix A. Supplementary data}

Supplementary data associated with this article can be found, in the online version, athttps://doi.org/10.1016/j.jmmm.2018.12.032.

\section{References}

[1] J. Angelkort, A. Senyshyn, A. Schönleber, S.V. Smaalen, Temperature-dependent neutron diffraction on $\mathrm{TiI}_{3}$, Z. Kristallogr. Cryst. Mater. 226 (2011) 640.

[2] H. Wang, V. Eyert, U. Schwingenschlögl, Electronic structure and magnetic ordering of the semiconducting chromium trihalides $\mathrm{CrCl}_{3}, \mathrm{CrBr}_{3}$, and $\mathrm{CrI}_{3}$, J. Phys.: Condens. Matter. 23 (2011) 116003

[3] H. Wang, F. Fan, S. Zhu, H. Wu, Doping enhanced ferromagnetism and induced halfmetallicity in $\mathrm{CrI}_{3}$ monolayer, EPL 114 (2016) 47001.

[4] H. Hillebrecht, T. Ludwig, G. Thiele, About trihalides with $\mathrm{TiI}_{3}$ chain structure: Proof of pair forming of cations in $\beta-\mathrm{RuCl}_{3}$ and $\mathrm{RuBr}_{3}$ by temperature dependent single crystal X-ray analyses, Z. Anorg. Allg. Chem. 630 (2004) 2199.

[5] Y. Zhou, H. Lu, X. Zu, F. Gao, Evidencing the existence of exciting half-metallicity in two-dimensional $\mathrm{TiCl}_{3}$ and $\mathrm{VCl}_{3}$ sheets, Sci. Rep. 6 (2016) 19407.

[6] X. Li, J. Yang, First-principles design of spintronics materials, Natl. Sci. Rev. 3 (2016) 365.

[7] A. Banerjee, C. Bridges, J. Yan, A. Aczel, L. Li, M. Stone, G. Granroth, M. Lumsden, Y. Yiu, J. Knolle, S. Bhattacharjee, Proximate kitaev quantum spin liquid behaviour in a honeycomb magnet, Nat. Mater. 15 (2016) 733.

[8] I. Tsubokawa, On the magnetic properties of a $\mathrm{CrBr}_{3}$ single crystal, J. Phys. Soc. Jpn. 15 (1960) 1664.

[9] J.M. Fletcher, W.E. Gardner, E.W. Hooper, K.R. Hyde, F.H. Moore, J.L. Woodhead, Anhydrous ruthenium chlorides, Nature 199 (1963) 1089.

[10] W. Baker, A. Janus, Magnetic properties of zirconium (iii) and hafnium (iii) iodides, J. Inorg. Nucl. Chem. 26 (1964) 2087.

[11] J. Lin, G.J. Miller, Dimensional diversity in transition metal trihalides, Inorg. Chem. 32 (1993) 1476.

[12] S. Troyanov, E. Snigireva, V. Rybakov, X-ray diffraction study of phase transition in $\alpha$-TiCl ., Zh, Neorg. Khimii 36 (1991) 1117.

[13] P. Miró, M. Audiffred, T. Heine, An atlas of two-dimensional materials, Chem. Soc. Rev. 43 (2014) 6537.

[14] H. Bengel, H.-J. Cantow, S. Magonov, H. Hillebrecht, G. Thiele, W. Liang, M.$\mathrm{H}$. Whangbo, Tip-force induced surface corrugation in layered transition metal trichlorides $\mathrm{MCl}_{3}$ ( $\mathrm{m}=\mathrm{ru}, \mathrm{mo}$, rh, ir), Surf. Sci. 343 (1995) 95.

[15] K. Plumb, J. Clancy, L. Sandilands, V.V. Shankar, Y. Hu, K. Burch, H.-Y. Kee, Y.J. Kim, $\alpha-\mathrm{RuCl}_{3}$ : a spin-orbit assisted mott insulator on a honeycomb lattice, Phys. Rev. B 90 (2014) 041112

[16] D. Weber, L.M. Schoop, V. Duppel, J.M. Lippmann, J. Nuss, B.V. Lotsch, Magnetic properties of restacked $2 \mathrm{~d}$ spin $1 / 2$ honeycomb $\mathrm{RuCl}_{3}$ nanosheets, Nano Lett. 16 (2016) 3578.

[17] J.F.D.Jr.C.E. Olson, Magnetization and resonance and optical properties of the ferromagnet $\mathrm{CrI}_{3}$, J. Appl. Phys. 36 (1965) 1259.

[18] B. Huang, G. Clark, E. Navarro-Moratalla, D. Klein, R. Cheng, K. Seyler, D. Zhong, E. Schmidgall, M. McGuire, D. Cobden, W. Yao, Layer-dependent ferromagnetism in a van der waals crystal down to the monolayer limit, Nature 546 (2017) 270.

[19] M. McGuire, J. Yan, P. Lampen-Kelley, A. May, V. Cooper, L. Lindsay, A. Puretzky, L. Liang, K. Santosh, E. Cakmak, S. Calder, B.C. Sales, High-temperature magnetostructural transition in van der Waals-layered $\alpha-\mathrm{MoCl}_{3}$, Phys. Rev. Mater. 1 (2017) 064001

[20] W.-B. Zhang, Q. Qu, P. Zhu, C.-H. Lam, Robust intrinsic ferromagnetism and half semiconductivity in stable two-dimensional single-layer chromium trihalides, J. Mater. Chem. C 3 (2015) 12457.

[21] J. He, S. Ma, P. Lyu, P. Nachtigall, Unusual dirac half-metallicity with intrinsic ferromagnetism in vanadium trihalide monolayers, J. Mater. Chem. C 4 (2016) 2518.

[22] A. Kuzubov, E. Kovaleva, M. Popova, A. Kholtobina, N. Mikhaleva, M. Visotin, A. Fedorov, Dft investigation of electronic structures and magnetic properties of halides family $\mathrm{MeHal}_{3}$ (me = ti, mo, zr, nb, ru, hal =cl, br, i) one dimensional structures, J. Magn. Magn. Mater. 440 (2017) 93.

[23] M.A. McGuire, Crystal and magnetic structures in layered, transition metal diha lides and trihalides, Crystals 7 (2017) 121.

[24] F. Iyikanat, M. Yagmurcukardes, R. Senger, H. Sahin, Tuning electronic and magnetic properties of monolayer $\alpha-\mathrm{RuCl}_{3}$ by in-plane strain, J. Mater. Chem. C 6 (2018) 2019.

[25] S. Sarikurt, Y. Kadioglu, F. Ersan, E. Vatansever, O.Ü. Aktürk, Y. Yüksel, Ü. Akınc1, E. Aktürk, Electronic and magnetic properties of monolayer $\alpha-\mathrm{RuCl}_{3}$ : a first-principles and monte carlo study, Phys. Chem. Chem. Phys. 20 (2018) 997.

[26] J. Liu, Q. Sun, Y. Kawazoe, P. Jena, Exfoliating biocompatible ferromagnetic crtrihalide monolayers, Phys. Chem. Chem. Phys. 18 (2016) 8777.

[27] M.A. McGuire, H. Dixit, V.R. Cooper, B.C. Sales, Coupling of crystal structure and magnetism in the layered, ferromagnetic insulator $\mathrm{CrI}_{3}$, Chem. Mater. 27 (2015) 612.

[28] C. Huang, J. Zhou, H. Wu, K. Deng, P. Jena, E. Kan, Quantum anomalous hall effect in ferromagnetic transition metal halides, Phys. Rev. B 95 (2017) 045113

[29] G. Kresse, J. Furthmüller, Efficient iterative schemes for ab initio total-energy calculations using a plane-wave basis set, Phys. Rev. B 54 (1996) 11169.

[30] G. Kresse, J. Furthmüller, Efficiency of ab-initio total energy calculations for metals and semiconductors using a plane-wave basis set, Comput. Mater. Sci. 6 (1996) 15.

[31] J.P. Perdew, J.A. Chevary, S.H. Vosko, K.A. Jackson, M.R. Pederson, D.J. Singh, C. Fiolhais, Atoms, molecules, solids, and surfaces: Applications of the generalized gradient approximation for exchange and correlation, Phys. Rev. B 46 (1992) 6671

[32] J.P. Perdew, K. Burke, M. Ernzerhof, Generalized gradient approximation made simple, Phys. Rev. Lett. 77 (1996) 3865.

[33] P.E. Blöchl, Projector augmented-wave method, Phys. Rev. B 50 (1994) 17953.

[34] G. Kresse, D. Joubert, From ultrasoft pseudopotentials to the projector augmentedwave method, Phys. Rev. B 59 (1999) 1758.

[35] H.J. Monkhorst, J.D. Pack, Special points for brillouin-zone integrations, Phys. Rev. B 13 (1976) 5188.

[36] A. Togo, I. Tanaka, First principles phonon calculations in materials science, Scr. Mater. 108 (2015) 1.

[37] N. Sivadas, M.W. Daniels, R.H. Swendsen, S. Oakamoto, D. Xiao, Magnetic ground state of semiconducting transition-metal trichalcogenide monolayers, Phys. Rev. B 91 (2015) 235425

[38] J. Angelkort, A. Schonleber, S. van Smaalen, Low-and high-temperature crystal structures of TiI3, J. Solid State Chem. 182 (2009) 525.

[39] C. Tan, X. Cao, X.-J. Wu, Q. He, J. Yang, X. Zhang, J. Chen, W. Zhao, S. Han, G.H. Nam, M. Sindoro, H. Zhang, Recent advances in ultrathin two-dimensional nanomaterials, Chem. Rev. 117 (2017) 6225.

[40] M. Ziatdinov, A. Banerjee, A. Maksov, T. Berlijn, W. Zhou, H. Cao, J. Yan, C. Bridges, D. Mandrus, S. Nagler, A. Baddorf, Atomic-scale observation of structural and electronic orders in the layered compound $\alpha-\mathrm{RuCl}_{3}$, Nat. Commun. 7 (2016) 13774.

[41] A. Kitaev, Anyons in an exactly solved model and beyond, Ann. Phys. 321 (2006) 2.

[42] M. Hermanns, I. Kimchi, J. Knolle, Physics of the Kitaev model: fractionalization, dynamic correlations, and material connections, Annu. Rev. Condens. Matter Phys. 9 (2018) 17.

[43] S.M. Winter, A.A. Tsirlin, M. Daghofer, J. van den Brink, Y. Singh, P. Gegenwart, R. Valenti, Models and materials for generalized Kitaev magnetism, J. Phys. Condens. Matter 29 (2017) 493002

[44] S. Trebst, Kitaev materials, arXiv (2017) 064430.

[45] L. Janssen, E.C. Andrade, M. Vojta, Magnetization processes of zigzag states on the honeycomb lattice: Identifying spin models for $\alpha-\mathrm{RuCl}_{3}$ and $\mathrm{Na}_{2} \mathrm{IrO}_{3}$, Phys. Rev. B 96 (2017) 064430

[46] L. Janssen, E.C. Andrade, M. Vojta, Honeycomb-lattice Heisenberg-Kitaev model in a magnetic field: Spin canting, metamagnetism, and vortex crystals, Phys. Rev. Lett. 117 (2016) 277202

[47] J. Chaloupka, G. Jackeli, G. Khaliullin, Kitaev-Heisenberg model on a honeycomb lattice: possible exotic phases in iridium oxides $\mathrm{A}_{2} \mathrm{IrO}_{3}$, Phys. Rev. Lett. 105 (2010) 027204 .

[48] J. Chaloupka, G. Jackeli, G. Khaliullin, Zigzag magnetic order in the iridium oxide $\mathrm{Na}_{2} \mathrm{IrO}_{3}$, Phys. Rev. Lett. 110 (2013) 097204.

[49] Y. Singh, S. Manni, J. Reuther, T. Berlijn, R. Thomale, W. Ku, S. Trebst, P. Gegenwart, Relevance of the Heisenberg-Kitaev model for the honeycomb lattice iridates $\mathrm{A}_{2} \mathrm{IrO}_{3}$, Phys. Rev. Lett. 108 (2012) 127203

[50] X. Yao, S. Dong, Topological triple-vortex lattice stabilized by mixed frustration in expanded honeycomb Kitaev-Heisenberg model, Sci. Rep. 6 (2016) 26750.

[51] E. Sela, H.C. Jiang, M.H. Gerlach, S. Trebst, Order-by-disorder and spin-orbital liquids in a distorted Heisenberg-Kitaev model, Phys. Rev. B 90 (2014) 035113.

[52] X. Yao, The expanded triangular Kitaev-Heisenberg model in the full parameter space, Phys. Lett. A 378 (2014) 2290. 
[53] C. Price, N.B. Perkins, Finite-temperature phase diagram of the classical KitaevHeisenberg model, Phys. Rev. B 88 (2013) 024410.

[54] C. Price, N.B. Perkins, Critical properties of the Kitaev-Heisenberg model, Phys Rev. Lett. 109 (2012) 187201

[55] S. Chandra, K. Ramola, D. Dhar, Classical heisenberg spins on a hexagonal lattice with Kitaev couplings, Phys. Rev. E 82 (2010) 031113.

[56] M.E.J. Newman, G.T. Barkema, Monte Carlo methods in statistical physics (199).

[57] J.A. Sears, M. Songvilay, K.W. Plumb, J.P. Clancy, Y. Qiu, Y. Zhao, D. Parshall, Y. J. Kim, Magnetic order in $\alpha-\mathrm{RuCl}_{3}$ : a honeycomb-lattice quantum magnet with strong spin-orbit coupling, Phys. Rev. B 91 (2015) 144420 . 